\title{
Interrelationships Among SADIE Indices for Characterizing Spatial Patterns of Organisms
}

\author{
Xiangming Xu and Laurence V. Madden
}

First author: East Malling Research, New Road, East Malling, Kent ME19 6BJ, UK; and second author: Department of Plant Pathology, Ohio State University, Wooster 44691. Accepted for publication 30 March 2005.

\begin{abstract}
Xu, X.-M., and Madden, L. V. 2005. Interrelationships among SADIE indices for characterizing spatial patterns of organisms. Phytopathology 95:874-883.

The SADIE (spatial analysis by distance indices) methodology for data analysis is a useful approach for quantifying the patterns of organisms (in terms of patches and gaps) and testing for randomness of the patterns. We investigated the interrelationship among key SADIE indices: index for distance to regularity for a data set $\left(I_{a}\right)$, a global measure of aggregation or clustering; the local clustering indices $\left(v_{i}\right.$ and $\left.v_{j}\right)$, scaled distances to regularity for each individual sampling unit; and the averages of $v_{i}$ and $v_{j}$

similar results. Overall differences in average $v_{i}$ and $\left|v_{j}\right|$ values, and between $I_{a}$ and these averages, decreased with increasing size of the sampling grid in a simulation study. This was because one component of $v_{i}$ and $v_{j}\left({ }_{i} Y\right)$-a measure of the distance to regularity under randomness for a given location (not a given count) — was found generally to vary little with location, except for locations near corners of the sampling grid. Nevertheless, because distance to regularity for individual observed counts was location-dependent, and this location effect varied with the observed counts value as well, a new-scaled index for each count $\times$ location combination may be warranted. The implications of these findings on epidemiological research are discussed.
\end{abstract} across all sampling units, which are additional global measures of aggregation. We demonstrated that $v_{i}$ and $v_{j}$ are mathematically related to $I_{a}$ and showed conditions when $I_{a}$ and mean local clustering indices give very
Additional keywords: index to regularity, sampling size.
Recently, a novel and innovative approach called SADIE (spatial analysis by distance indices) was developed to quantify spatial patterns of organisms for either spatially referenced sampling units $(7,8)$ or individuals $(7)$. The methodology has been successfully used in several epidemiological studies (11-13). The basis of SADIE for spatially referenced sampling units, in its most commonly used form, is to quantify pattern by the total distance that individuals (e.g., diseased plants) must be moved between sampling units so that the data are as regular as possible (i.e., there is the same number of individuals in each sampling unit). The degree of nonrandomness is quantified by comparing the distance to regularity for the observed data set with distance to regularity for rearrangements of the observed data. One of the SADIE statistics, $I_{a}$, is defined as $D / E_{a}$, where $D$ is the distance moved to achieve the regular pattern for the observed data, and $E_{a}$ is the arithmetic mean distance to regularity for the $m$ randomized samples.

The SADIE methodology was expanded further to define and analyze the spatial pattern of a data set (10). The contribution of each sampling-unit count to the pattern (in terms of "patches" and "gaps") is quantified by determining a scaled and unitless clustering index for each sampling unit using the observed and randomized data. The clustering indices for sampling units with observed counts greater and less than the mean are indicated by $v_{i}$ (a positive value) and $v_{j}$ (a negative value), respectively. The indices $v_{i}$ and $v_{j}$ measure the respective degree to which a sampling unit contributes to clustering as a member of a patch and as a member of a gap. Mean $v_{i}\left(\bar{v}_{i}\right)$ and $v_{j}\left(\bar{v}_{j}\right)$ values for a data set are to be used as measures of the degree of nonrandomness in addition to

Corresponding author: X.-M. Xu; E-mail address: Xiangming.Xu@emr.ac.uk

DOI: 10.1094/PHYTO-95-0874

(C) 2005 The American Phytopathological Society
$I_{a}$. Recently, the SADIE method has been extended to assess the spatial association of two species $(9,15)$ by correlating local association indices calculated from the individual sampling-unit clustering indices for each species. The SADIE methodology has evolved into a more graphical analysis of spatial patterns of local clustering and associating indices, rather than focusing solely on single indices of aggregation and association (10).

SADIE methodology has recently been used in several epidemiological investigations (11-13). However, the interpretation of and the interrelationships among the SADIE statistics are not always apparent because SADIE is based explicitly on randomization results for determining all indices and significance tests. For instance, we recently found that $I_{a}$ has near-perfect linear correlation with $\bar{v}_{i}$ and $\bar{v}_{j}$ in a simulation study of plant disease epidemics (17). Indeed, the absolute values of these three indices are nearly equal, suggesting that just one of the indices may be sufficient to represent the pattern. Most importantly, these indices depended critically on initial epidemic conditions: number of initial infected plants and their spatial configuration (17). Although Perry et al. (10) now recommend the use of $v_{i}$ - and $v_{j}$-based statistics for quantifying patterns in addition to the $I_{a}$ statistic, it is still common for others to simply report $I_{a}$ (and associated significance level).

In order to fully exploit the value of SADIE in comparing spatial results from different experiments, and hence, inferring biological/ecological differences between experiments, we need to understand the relationships of SADIE statistics with underlying biological and physical processes. Only then may we be able to ascertain to what extent the differences among statistics from different studies reflect biological/ecological differences of interest. SADIE indices previously were shown to be sensitive to absolute (and not just relative) positions of patches (16). Since $v_{i}$ and $v_{j}$ have been given paramount importance in graphical representations of the spatial system under investigation and in quantifying 
species association $(9,10,15)$, it is important to understand how these indices depend on physical properties of the spatial system being studied.

In this study, we aim to investigate the basis of the observed close relationship among the SADIE indices $\left(I_{a}, \bar{v}_{i}\right.$, and $\left.\bar{v}_{j}\right)$ and the relationship of local clustering indices with physical properties of the system. First, we show the relationship among SADIE indices using results from two recently published experimental investigations. Then, we show the mathematical interrelationships among the three SADIE indices. Finally, we shall use a few artificially generated data sets to illustrate the interrelationships among $I_{a}, \bar{v}_{i}$, and $\bar{v}_{j}$, and the properties of clustering indices in relation to several physical factors often encountered in epidemiological experiments, including sampling sizes, grid index system, and number of randomizations. SADIE statistics were calculated using the software (SADIESHELL) provided by J. N. Perry of Rothamsted Research, England, UK.

SADIE methodology-description of the method. Table 1 provides the notation and definition of important variables used to define SADIE statistics. The number or count of individuals in a sampling unit is given by $x$, and the mean count for the data set by $\bar{x}$. In SADIE methodology, sampling units with counts larger than the mean are donors, designated with an $i$ subscript, in that individuals are moved out of these units in determining the total moves to regularity. Similarly, sampling units with counts smaller than the mean are receivers, designated with a $j$ subscript, in that individuals moved out of the donor units are eventually placed in these units. For donor unit $i$, the total outflow of individuals is given by $x_{i}-\bar{x}$. Likewise, for receiver unit $j$, total inflow of individuals is given by $\bar{x}-x_{j}$. There are a total of $n_{i}$ donor $(i=1, \ldots$, $n_{i}$ ) sampling units and $n_{j}$ receiver $\left(j=1, \ldots, n_{j}\right)$ sampling units. The specific moves to regularity are determined by a transportation algorithm $(7,8)$, a computationally intensive procedure.

For donor unit $i$, at position $\left(a_{i}, b_{i}\right)$, the outflow to the $j$ th of $n_{j}$ receiver units, $j=1, \ldots, n_{j}$, at position $\left(a_{j}, b_{j}\right)$, is denoted as $V_{i j}$. The distance of this flow, denoted as $d_{i j}$, is $\sqrt{\left(a_{i}-a_{j}\right)^{2}+\left(b_{i}-b_{j}\right)^{2}}$. The sum of the $V_{i j}$ over all receiver units $\left(\Sigma_{j} V_{i j}\right)$ for this donor unit $i$ is simply $x_{i}-\bar{x}$. The average distance of outflow from unit $i$ to all receivers, weighted by the magnitude of each individual flow, is denoted as $Y_{i}$,

$$
Y_{i}=\frac{\sum_{j} d_{i j} V_{i j}}{\sum_{j} V_{i j}}=\frac{D_{i}}{x_{i}-\bar{x}}
$$

It should be noted that the global distance to regularity for the entire data set, used in determining $I_{a}$, is $D=\sum_{i} D_{i}$.

A dimensionless index of clustering, $v$, was then obtained from $Y_{i}$ and may be used to compare between different units and between different sets of data. For outflows, a standardized and dimensionless index of clustering, $v_{i}$, is given by Perry et al. (10) as

$$
v_{i}=\frac{Y_{i o} Y}{{ }_{i} Y_{c} Y}
$$

where ${ }_{c} Y$ and ${ }_{i} Y$ are expected values based on the randomizations as described below. The individual (original) counts are kept track of during the randomizations, and their average distance of outflow calculated for each randomization $\left(Y_{i, k} ; k=1, \ldots, m\right) .{ }_{c} Y$ is the average outflow distance for the observed count, $x_{i}$, across all the randomizations, i.e.,

$$
{ }_{c} Y=\sum_{k=1}^{m} Y_{i, k} / m
$$

Similarly, instead of following the count through its randomizations to other units, the individual location for the $i$ th unit $\left(a_{i}, b_{i}\right)$ is kept track of, which will contain any one of the original counts in a given randomization. The absolute value of the average flow distance for each location is determined in each randomization $\left({ }_{k, i} Y ; k=1, \ldots, m\right)$ and the average for this location across all randomizations for unit $i$ is denoted as ${ }_{i} Y$, i.e.,

$$
{ }_{i} Y=\sum_{k=1}^{m}{ }_{k, i} Y / m
$$

Hence, ${ }_{i} Y$ is the expected value of the average absolute flow distance for the $i$ th unit, under the assumption of a random arrangement of the observed counts among the sample units. Furthermore, the average absolute value of ${ }_{c} Y$ for a data set over all donor and receiver units, which is equal to the average value of ${ }_{i} Y$ over all the units, is denoted ${ }_{o} Y$. The mean clustering index for donor units, averaged across the $n_{i}$ donor units, is then denoted $\bar{v}_{i}=\left(\sum_{i} v_{i}\right) / n_{i}$.

For inflows, $v_{j}$ is defined similarly, again with the convention that it is negative in sign. As a general rule, large values of $v_{i}$ (or $\bar{v}_{i}$ for the entire data set) $\approx>1.5$ indicate patchiness; large negative values of $v_{j}\left(\right.$ or $\left.\bar{v}_{j}\right) \approx<-1.5$ indicate membership to a gap; and values close to unity indicate a random placement of that unit in relation to others nearby (10). Note, $E_{a},{ }_{i} Y,{ }_{c} Y$, and ${ }_{o} Y$ are all defined, theoretically, as expected values for a random pattern (10). More formally, their calculated values are expected values only with an infinite number of randomizations. With the reality of a finite number of randomizations, the calculations give estimates of the true expected values.

Experimental data on the relationship of $\boldsymbol{I}_{\boldsymbol{a}}, \overline{\boldsymbol{v}}_{\boldsymbol{i}}$, and $\overline{\boldsymbol{v}}_{\boldsymbol{j}}$. We have obtained two sets of data on $I_{a}, \bar{v}_{i}$, and $\bar{v}_{j}$ from two recently published studies $(11,12)$ to demonstrate the empirical relationship between these measures of nonrandomness. Although neither paper reported the $\bar{v}_{i}$ and $\bar{v}_{j}$ values, we obtained these from the authors and used these with the reported $I_{a}$ values (with corrections for typesetting errors). Figure 1 shows the pair-wise relationships among the absolute values of $I_{a}, \bar{v}_{i}$, and $\bar{v}_{j}$ for these

\begin{tabular}{|c|c|}
\hline Symbol & Definition \\
\hline$x_{i}$ & $\begin{array}{l}\text { Individual donor unit counts }\left(x_{i}>\bar{x} \text {, where } \bar{x} \text { is the mean for a data set). Similarly, an index } j \text { is used for a receiver unit }\left(x_{j}<\bar{x}\right) \text {. For simplicity, }\right. \\
\text { only symbols for donors (index } i \text { ) are given below }\end{array}$ \\
\hline$D_{i}$ & Overall distance moved to achieve the regular pattern for donor unit $i$ \\
\hline$E_{a}$ & Arithmetic mean distance to regularity for the a number of permutations for a whole data set \\
\hline$I_{a}$ & An index to regularity $=D / E_{a}$ \\
\hline$Y_{i}$ & Average distance of outflow from unit $i$ to all receivers $=D_{i} /\left(x_{i}-\bar{x}\right)$ \\
\hline$u_{i}$ & A dimensionless index, defined as $u_{i}=Y_{i} /_{c} Y$ \\
\hline$w_{i}$ & A dimensionless index, defined as $w_{i}={ }_{o} Y I_{i} Y$ \\
\hline$v_{i}$ & A dimensionless clustering index for each counts data at a particular location, defined as $v_{i}=\left(Y_{i o} Y\right) /\left({ }_{i} Y_{c} Y\right)=u_{i} \cdot w_{i}$ \\
\hline $\bar{v}_{i}$ & Average of clustering indices, $v_{i}$, over all donor units for a whole data set, equivalent to average of weighted $u_{i}$, with weights given by $w_{i}$ \\
\hline
\end{tabular}
two data sets. The Pearson product-moment correlation coefficient was $>0.9(P<0.001)$ for both data sets.

TABLE 1. Notation and definition of key variables 
Mathematical relationship of $\overline{\boldsymbol{v}}_{\boldsymbol{i}}$ and $\overline{\boldsymbol{v}}_{j}$ with $\boldsymbol{I}_{a}$. Assuming we have $m$ randomizations, then ${ }_{c} Y$ can be written as

$$
{ }_{c} Y=\frac{\sum_{k=1}^{m} Y_{i, k}}{m}=\frac{\sum_{k=1}^{m} D_{i, k}}{m\left(x_{i}-\bar{x}\right)}
$$

where $D_{i, k}$ is the total outflow distance for the sampling counts, $x_{i}$, in the $k$ th randomization. Combining this with the equation above for $Y_{i}$, one obtains

$$
v_{i}=\frac{Y_{i o} Y}{{ }_{i} Y Y}=\frac{D_{i} /\left(x_{i}-\bar{x}\right)}{\sum_{k=1}^{m} D_{i, k} / m\left(x_{i}-\bar{x}\right)} \cdot \frac{{ }_{o} Y}{{ }_{i} Y}=\frac{D_{i}}{\sum_{k=1}^{m} D_{i, k} / m} \cdot \frac{{ }_{o} Y}{{ }_{i} Y}=\frac{D_{i}}{\bar{D}_{i}} \cdot \frac{{ }_{o} Y}{{ }_{i} Y}
$$

where

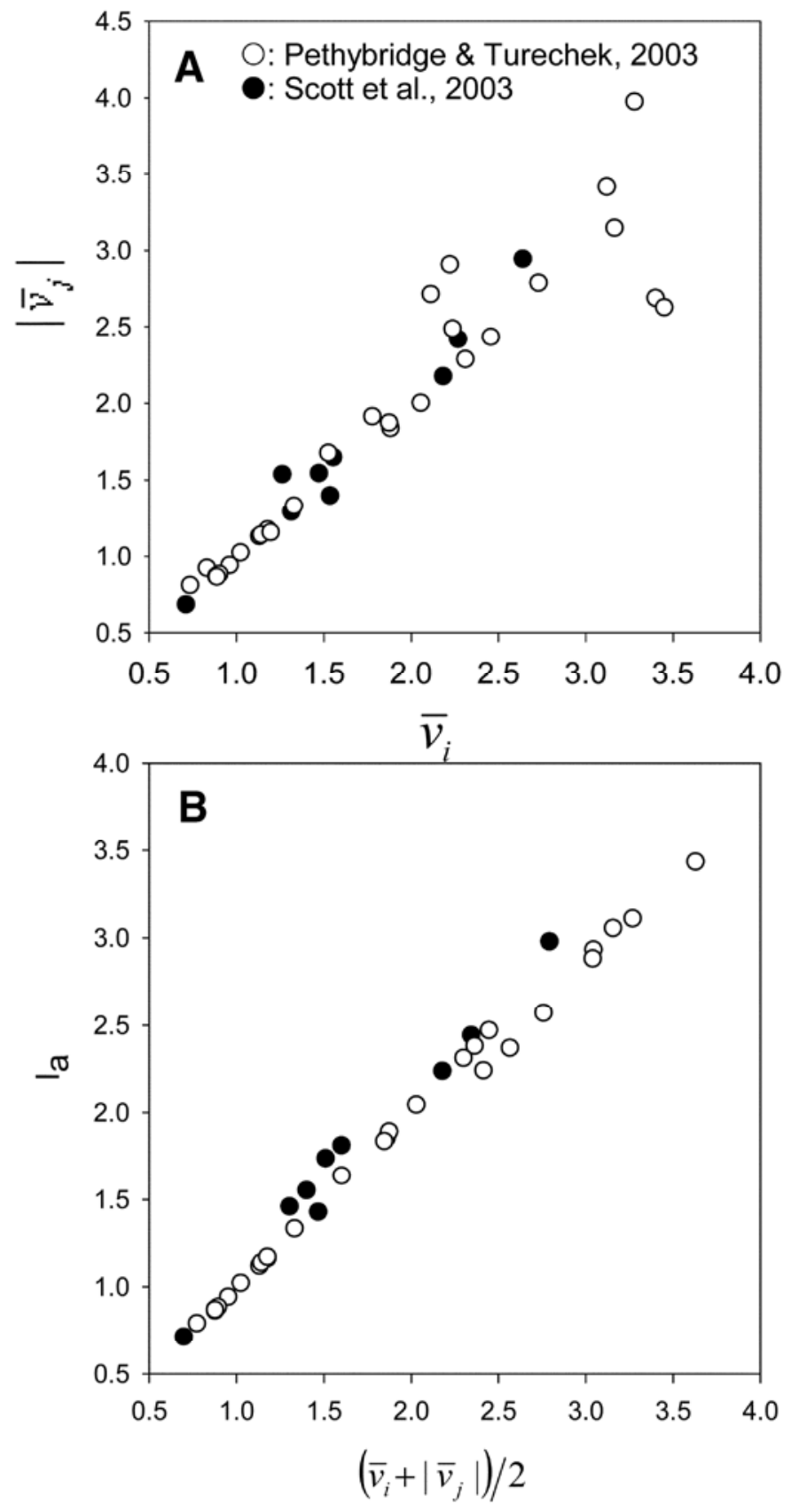

Fig. 1. Plot of $\bar{v}_{i}$ against $\left|\bar{v}_{j}\right|$ and $\left(\bar{v}_{i}+\left|\bar{v}_{j}\right|\right) / 2$ against $I_{a}$ from two experimental studies: open circle, Pethybridge and Turechek (11); and filled circle, Scott et al. (12). Table 1 provides notation and symbols.

$$
\bar{D}_{i}=\sum_{k=1}^{m} D_{i, k} / m
$$

is the average total distance to regularity across the randomizations for the observed counts, $x_{i}$. Moreover,

$$
\sum_{i=1}^{n_{i}} \overline{D_{i}}
$$

is identical to $E_{a}$, the average total distance to regularity for all randomizations. To simplify the following presentations, we can define $u_{i}$ as

$$
u_{i}=\frac{D_{i}}{\overline{D_{i}}}=\frac{Y_{i}}{{ }_{c} Y}
$$

for the $i$ th sampling unit. $u_{j}$ is defined similarly for the sampling units with counts less than the mean. Compared to the $I_{a}$ definition, $u_{i}$ is equivalent to the $I_{a}$ for the individual sampling counts data. Hence, from equation $4, v_{i}$ is equivalent to the $I_{a}$ value for the individual sampling counts data $\left(u_{i}\right)$ weighted by a factor $w_{i}$ $\left(w_{i}={ }_{o} Y I_{i} Y\right)$ depending on its spatial location; that is,

$$
v_{i}=\frac{Y_{i}}{{ }_{c} Y} \cdot \frac{{ }_{o} Y}{{ }_{i} Y}=\frac{Y_{i}}{{ }_{c} Y} \cdot w_{i}=u_{i} \cdot w_{i}
$$

Given that there are $n_{i}$ outflow units in a data set, $\bar{v}_{i}$, an average of this weighted $u_{i}$ for each outflow unit is written as

$$
\bar{v}_{i}=\frac{\sum_{i=1}^{n_{i}} v_{i}}{n_{i}}=\frac{\sum_{i=1}^{n_{i}}\left(u_{i} \cdot w_{i}\right)}{n_{i}}=\frac{\sum_{i=1}^{n_{i}}\left(\frac{D_{i}}{\bar{D}_{i}} \cdot w_{i}\right)}{n_{i}}
$$

Similarly, with $n_{j}$ inflow units, one can write

$$
\bar{v}_{j}=\frac{\sum_{j=1}^{n_{j}} v_{j}}{n_{j}}=\frac{\sum_{j=1}^{n_{j}} u_{j} \cdot w_{j}}{n_{j}}=\frac{\sum_{j=1}^{n_{j}}\left(\frac{D_{j}}{\bar{D}_{j}} \cdot w_{j}\right)}{n_{j}}
$$

For the whole data set, $I_{a}$ is then

$$
I_{a}=\frac{D}{E_{a}}=\frac{\sum_{i=1}^{n_{i}} D_{i}}{\sum_{i=1}^{n_{i}} \bar{D}_{i}}=-\frac{\sum_{j=1}^{n_{j}} D_{j}}{\sum_{j=1}^{n_{j}} \overline{D_{j}}}
$$

where

$$
\sum_{i=1}^{n_{i}} D_{i}=-\sum_{j=1}^{n_{j}} D_{j}=D
$$

Therefore, the global $I_{a}$ is related to the mean clustering index via the distance moved for each individual sampling unit to achieve a regular distribution, whereas the mean clustering index is an average of location-weighted $I_{a}$, i.e., $u_{i}$ or $u_{j}$, over all outflow or inflow sampling units.

\section{THEORY AND APPROACHES}

The key to understanding the properties of individual clustering indices and the relationships among $I_{a}, \bar{v}_{i}$, and $\bar{v}_{j}$ is to understand the properties of ${ }_{c} Y$ and ${ }_{i} Y$. In this study, we generated a number of data sets to investigate the properties of ${ }_{c} Y$ and ${ }_{i} Y$ in relation to nonbiological factors, including sampling grid size, number of randomizations, frequency distribution, and spatial location of a specific count. All statistical analyses were carried out using Genstat (VSN International, Oxford) (6).

Data generation. We generated two data sets for each of three sampling sizes: $5 \times 5,10 \times 10$, and $20 \times 20$. One was drawn from a Poisson distribution with a mean $(\mu)$ of 50 . The other was from a beta-binomial distribution with the parameters of $\alpha=5$ and $\beta=$ 5 , and a sample unit size $\left(n_{s u}\right)$ of 100 . This combination of parameters gave a mean the same as the Poisson distribution, $\mu=$ 
$n_{s u} \alpha /(\alpha+\beta)$. The heterogeneity parameter of the beta-binomial was $1 /(\alpha+\beta)=0.1$. The exact original spatial location of each count is irrelevant since ${ }_{c} Y$ and ${ }_{i} Y$ are averages over all randomizations and hence only dependent on the actual counts and the grid system used.

From these initial six data sets, we generated an additional 18 data sets. Six of the eighteen were obtained simply by multiplying the spatial grid indices by five, except the original indices of 1 (i.e., the resulting spatial location indices are $(1,1),(1,5)$, and $(1,10) \ldots$. instead of $(1,1),(1,2)$, and $(1,3)$, etc.). This extended the sampling coverage (i.e., physical area) without changing the counts. Another six sets were obtained by modifying original counts data to increase the data range by the following rule:

$$
z_{i}=x_{i}\left(\frac{x_{i}}{\bar{x}}\right)^{2}
$$

where $z_{i}$ and $x_{i}$ are the transformed and original counts data, respectively. This transformation is used for two reasons; in addition to the increased data range, the transformation will also generate data that are skewed to the right. Of course, the newly obtained data would not be expected to conform to either a Poisson or a beta-binomial distribution. However, for ease of reference, we still use the distribution name to refer to the origin of the data sets. In the final six data sets, both original data and spatial indices were replaced by the modified data with an increased range and the expanded spatial indices, respectively.

Properties of ${ }_{c} \boldsymbol{Y}$ and ${ }_{i} \boldsymbol{Y}$. For each of the 24 data sets, we used the SADIE software to calculate the ${ }_{c} Y$ and ${ }_{i} Y$ values using the 39 , 390 , and 3,900 randomizations. Through the 24 generated data sets, we were able to investigate the effects of the following factors-data distribution, sampling size and index system, range of counts data, and number of randomizations - on ${ }_{c} Y$ and ${ }_{i} Y$.

We calculated the coefficient of variation (CV) for each generated set of ${ }_{c} Y$ and ${ }_{i} Y$. Analysis of variance (ANOVA) was used to determine the magnitude of the effects of the factors on these $\mathrm{CVs}$. Then, we investigated the relationship between ${ }_{c} Y$ and count $x_{i}$, and between ${ }_{i} Y$ and grid position (location; $\left.a_{i}, b_{i}\right)$. By definition, there is no relationship between ${ }_{c} Y$ and grid position (since ${ }_{c} Y$ is defined only in terms of the count, not location) nor between ${ }_{i} Y$ and $x_{i}$ (since ${ }_{i} Y$ is defined only in terms of the location, not the count). In all subsequent analyses, we use $x$ to represent observed counts data when all the data are included in analysis. Regression analysis was used to quantify these relationships. In the analysis of ${ }_{c} Y$ and $x_{i}$, four different models were used. Model 1 assumed a common relationship for both outflow and inflow units, whereas the other three models assumed a different relationship for outflow and inflow. Namely, model 1 is of the following form:

$$
\left|{ }_{c} Y\right|=a+b|x-\bar{x}|
$$

In model 2, a common slope $(b)$ but different intercept $(a)$ were assumed for outflow and inflow; in model 3, a common intercept (a) but different slope $(b)$ were assumed; and in model 4, different slopes as well as intercepts were assumed.

Relationships among SADIE statistics. Six data sets were used to explore the relationship of $u_{i}$ (i.e., individual samplingunit $I_{a}$ ) with $v_{i}$ and $v_{j}$, and of $I_{a}$ with $\bar{v}_{i}$ and $\bar{v}_{j}$ : original $5 \times 5$, $10 \times 10$, and $20 \times 20$ beta-binomial data sets and their corresponding data sets with larger range. In addition, a set of $3 \times 3$ counts data was generated from a beta-binomial distribution $(\alpha=\beta=5$ and $n_{s u}=100$ ) to further investigate the effect of small sampling sizes on the relationships among SADIE indices.

For each of the above seven data sets, we conducted 500 random permutations to generate 500 different spatial data sets but with the same counts data. For each permutation, the total distance to regularity $(D)$ was obtained. Theoretically, the $E_{a},{ }_{c} Y$, and ${ }_{i} Y$ values obtained from the SADIE randomizations for these 500 permutated data sets of each of the seven original data sets should be the same since these are functions of the frequency distribution of the original counts and not of the spatial locations of the counts. However, the estimated values of $E_{a},{ }_{c} Y$, and ${ }_{i} Y$ obtained from the SADIE software vary between the 500 permutated data sets, because the number of randomizations (even when $m$ is $>3,000)$ typically is not large enough to capture all the possible arrangements of the counts. So, in order to eliminate the effects of variation in estimates of $E_{a},{ }_{c} Y$, and ${ }_{i} Y$ among the permutations on the estimates of $I_{a}, u_{i}, v_{i}$, and $v_{j}$, only one common set of $E_{a},{ }_{c} Y$, and ${ }_{i} Y$ was used for each of the seven original data sets. In addition, ${ }_{c} Y$ values should be the same for all sampling units with the same counts data within a data set. However, as currently implemented in the SADIE software, this is not the case because the ${ }_{c} Y$ values are not averaged over all sampling units with same counts within a data set. As demonstrated herein, ${ }_{c} Y$ can be accurately estimated for a given data set with a regression model (model 2 where a common slope $(b)$ but different intercept $(a)$ were assumed for outflow and inflow in equation 11). Thus for the above two reasons, instead of using the ${ }_{c} Y$ value directly from randomizations, this regression model was used to estimate ${ }_{c} Y$ using the one common set of ${ }_{c} Y$ values, as described previously, for each of these seven data sets. For all permutated data sets, 3,900 randomizations were used to calculate SADIE statistics. For each of the seven original data sets, we used regression analysis to determine the relationships between 500 pairs of $\bar{v}_{i}$ and $\left|\bar{v}_{j}\right|$, and $\left(\bar{v}_{i}+\left|\bar{v}_{j}\right|\right) / 2$ and $I_{a}$, i.e., one pair from each permutation.

Effects of spatial location on $v_{i}$ and $v_{j}$. Because of the intensive computation required, we only used the original $5 \times 5$ data set generated with a beta-binomial distribution to investigate the effect of spatial locations of individual counts data on $Y_{i}$, hence $v_{i}$ and $v_{j}$. We generated a further 1,000 permutations for each of four situations where the maximum count $\left[\max \left(x_{i}\right)=78\right]$ was forced to be placed in a specified location (but the other counts were randomly placed, as before, for each permutation). The maximum count was placed at either $(1,1)$, i.e., at the corner; $(1,3)$, i.e., on the edge, half-way from the corners; $(3,3)$, i.e., in the center; and randomly (the nominal approach). For each of the above permutations, we calculated $Y_{i}$ for the count $x_{i}=78$ using the SADIE software. The cumulative frequency distribution of this $Y_{i}$ across the 1,000 permutations was then compared between the four locations. This procedure was repeated for two more counts: $x_{j}=$ 15 (minimum) and $x_{i}=52$ (close to the mean count) in the same $5 \times 5$ data set. In calculating $v_{i}$ and $v_{j}$ for all permutations at all positions for the three selected counts, ${ }_{o} Y$ and ${ }_{i} Y$ values from the 3,900 randomizations of the corresponding original data set, as described above, were used.

\section{RESULTS}

Properties of ${ }_{c} \boldsymbol{Y}$ and ${ }_{i} \boldsymbol{Y}$. Table 2 shows a partial summary of the effects of physical factors on variation of ${ }_{i} Y$ and ${ }_{c} Y$. ANOVA of the $\mathrm{CV}$ values showed the importance of the number of randomizations to the resulting indices. For the CVs, more than $75 \%$ of the variation was due to the main effects of the number of randomizations and its interaction with other factors. As expected, the greater the number of randomizations, the smaller the CV.

In all the following analyses, only results from 3,900 randomizations were used. Overall, the $\mathrm{CV}$ of ${ }_{i} Y$ was about half of that of ${ }_{c} Y$. Average $\mathrm{CV}$ for ${ }_{i} Y$ was about $3.6 \%$. ${ }_{i} Y$ was significantly related to spatial location (Fig. 2). In particular, those units near (but not at) the corners had much smaller ${ }_{i} Y$ values than other units. Interestingly, the corner units had much greater ${ }_{i} Y$ values than those nearby units (Fig. 2). On the other hand, units on the edges but not near the corners had greater ${ }_{i} Y$ values for sampling sizes of $10 \times 10$ and $20 \times 20$. For the sampling size of $5 \times 5$, the corner units had the greatest ${ }_{i} Y$ values, whereas those units on edges but not at the corner had the lowest ${ }_{i} Y$. If those units at or near the corners were excluded, the $\mathrm{CV}$ was reduced to about $2.5 \%$. A quadratic model of the spatial locations accounted for about $60 \%$ 
of the variation in ${ }_{i} Y$. As expected, ${ }_{i} Y$ was not related to the actual counts (data not shown). The $\mathrm{CV}$ of ${ }_{i} Y$ did not vary much with sampling size, range of counts data, and grid indices, although it appears that larger sampling size led to a smaller CV (Table 2).

Regression analysis showed that ${ }_{c} Y \mid$ was almost perfectly linearly related to $|x-\bar{x}|$ (Fig. 3). Increasing the numbers of randomizations led to a significant increase in the accuracy of this regression model, as seen from the percent variance accounted for $\left(R_{a}^{2}\right.$; Table 2), particularly for the large sampling sizes. The precise form of this linear relationship could, however, differ between inflow and outflow units; this was particularly so for the data set with large range and large sampling size (Table 2). That is, model 1 (which specifies a common relationship for outflow and inflow) was often inferior to the other models (in terms of percent variance accounted for) for the $10 \times 10$ and $20 \times 20$ grids with large data range. Interestingly, results for the $5 \times 5$ sampling size differed from those for $10 \times 10$ and $20 \times 20$ sizes for the Poissongenerated data. For the cases in which there were significant differences in the linear relationship between inflow and outflow units, the differences appeared to result mainly from the differences in the intercept rather than the slope (Table 2). Namely, where significant differences existed in model fits, model 2 often resulted in a better fit than model 3 and accounted for a similar amount of variance as model 4 .
Relationships among SADIE statistics. The Pearson productmoment correlation of $u_{i}$ (or $\left|u_{j}\right|$ ) with $v_{i}$ (or $\left|v_{j}\right|$ ) was very high and, on average, increased with increasing sampling size. For the $3 \times 3$ sampling size, there were a considerable number (22 of 500 ) of permutations where the correlation was $<0.85$, although for most cases (342 of 500) it was $>0.95$. For the $5 \times 5$ sampling size, all correlations were $>0.95$, whereas for the $10 \times 10$ and $20 \times$ 20 , all correlations were $>0.99$. There were no discernible differences in the correlation between different data sets of the same sampling size with different data ranges.

Figure 4A plots the differences between $\bar{v}_{i}$ and $\left|\bar{v}_{j}\right|$ for four selected data sets. Clearly, the difference between $\bar{v}_{i}$ and $\left|\bar{v}_{j}\right|$ decreased with increasing sampling size. The average differences between $\bar{v}_{i}$ and $\left|\bar{v}_{j}\right|$ were $8.6,7.5,4.1$, and $2.7 \%$ of their averages for sampling sizes of $3 \times 3,5 \times 5,10 \times 10$, and $20 \times 20$, respectively. The average $\bar{v}_{i}$ and $\left|\bar{v}_{j}\right|$ values were close to 1 , as expected. Furthermore, there were similar numbers of permutations with $\bar{v}_{i}>\left|\bar{v}_{j}\right|$, as those with $\bar{v}_{i}<\left|\bar{v}_{j}\right|$ (Fig. 4A). The effect of sampling size on the relationship between $\bar{v}_{i}$ and $\left|\bar{v}_{j}\right|$ is further demonstrated in the regression analysis (Table 3 ). The smaller the sampling size, the smaller the percentage of variance accounted for by the linear model. Similarly, the smaller the sampling size, the greater the intercept and the greater the slope deviated from 1.0

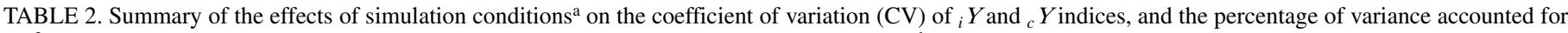
$\left(R_{a}^{2}\right.$, the adjusted coefficient of determination) by four models relating ${ }_{c} Y$ to counts per quadrat ${ }^{b}$

\begin{tabular}{|c|c|c|c|c|c|c|c|c|c|c|c|c|c|c|c|}
\hline \multirow{3}{*}{$\begin{array}{l}\text { Sampling } \\
\text { size }\end{array}$} & \multirow{3}{*}{$\begin{array}{c}\text { Data } \\
\text { range }\end{array}$} & \multirow{3}{*}{$\begin{array}{l}\text { Grid } \\
\text { index }\end{array}$} & \multirow[b]{3}{*}{ Randomizations } & \multicolumn{6}{|c|}{ Poisson distribution } & \multicolumn{6}{|c|}{ Beta-binomial distribution } \\
\hline & & & & \multicolumn{2}{|c|}{$\mathrm{CV}$} & \multicolumn{4}{|c|}{$R_{a}{ }^{2}$ for each model } & \multicolumn{2}{|c|}{$\mathrm{CV}$} & \multicolumn{4}{|c|}{$R_{a}^{2}$ for each model } \\
\hline & & & & ${ }_{c} Y$ & ${ }_{i} Y$ & 1 & 2 & 3 & 4 & ${ }_{c} Y$ & ${ }_{i} Y$ & 1 & 2 & 3 & 4 \\
\hline \multirow[t]{12}{*}{$5 \times 5$} & \multirow[t]{6}{*}{1} & \multirow[t]{3}{*}{1} & 39 & 9.5 & 7.6 & 0.65 & 0.73 & 0.70 & 0.73 & 8.3 & 6.6 & 0.58 & 0.70 & 0.62 & 0.72 \\
\hline & & & 390 & 9.2 & 4.5 & 0.86 & 0.96 & 0.92 & 0.96 & 9.1 & 4.2 & 0.85 & 0.95 & 0.92 & 0.95 \\
\hline & & & 3,900 & 8.8 & 4.1 & 0.87 & 0.97 & 0.93 & 0.97 & 8.3 & 3.7 & 0.87 & 0.99 & 0.94 & 0.99 \\
\hline & & \multirow[t]{3}{*}{2} & 39 & 11.7 & 5.5 & 0.71 & 0.76 & 0.76 & 0.77 & 9.2 & 6.2 & 0.46 & 0.65 & 0.49 & 0.71 \\
\hline & & & 390 & 9.3 & 4.8 & 0.83 & 0.91 & 0.89 & 0.91 & 9.1 & 4.9 & 0.86 & 0.95 & 0.93 & 0.95 \\
\hline & & & 3,900 & 8.9 & 4.2 & 0.87 & 0.96 & 0.93 & 0.96 & 8.5 & 3.9 & 0.88 & 0.99 & 0.95 & 0.99 \\
\hline & \multirow[t]{6}{*}{2} & \multirow[t]{3}{*}{1} & 39 & 8.9 & 6.4 & 0.81 & 0.81 & 0.81 & 0.81 & 9.3 & 7.6 & 0.56 & 0.72 & 0.66 & 0.72 \\
\hline & & & 390 & 8.1 & 3.4 & 0.96 & 0.96 & 0.96 & 0.96 & 9.0 & 4.4 & 0.77 & 0.96 & 0.91 & 0.96 \\
\hline & & & 3,900 & 8.2 & 3.5 & 0.98 & 0.98 & 0.98 & 0.98 & 8.3 & 3.2 & 0.80 & 0.99 & 0.95 & 1.00 \\
\hline & & \multirow[t]{3}{*}{2} & 39 & 10.1 & 5.3 & 0.66 & 0.66 & 0.66 & 0.67 & 10.7 & 6.7 & 0.65 & 0.73 & 0.77 & 0.77 \\
\hline & & & 390 & 8.6 & 3.7 & 0.96 & 0.96 & 0.96 & 0.96 & 8.2 & 3.7 & 0.75 & 0.94 & 0.89 & 0.94 \\
\hline & & & 3,900 & 8.3 & 3.6 & 0.98 & 0.99 & 0.98 & 0.99 & 8.2 & 3.2 & 0.78 & 1.00 & 0.93 & 1.00 \\
\hline \multirow[t]{12}{*}{$10 \times 10$} & \multirow[t]{6}{*}{1} & \multirow[t]{3}{*}{1} & 39 & 10.3 & 6.8 & 0.50 & 0.50 & 0.50 & 0.50 & 11.1 & 7.7 & 0.54 & 0.55 & 0.54 & 0.55 \\
\hline & & & 390 & 7.6 & 3.4 & 0.90 & 0.90 & 0.90 & 0.90 & 7.8 & 3.5 & 0.91 & 0.92 & 0.92 & 0.92 \\
\hline & & & 3,900 & 7.2 & 3.0 & 0.99 & 0.99 & 0.99 & 0.99 & 7.0 & 2.7 & 0.97 & 0.99 & 0.98 & 0.99 \\
\hline & & \multirow[t]{3}{*}{2} & 39 & 9.5 & 7.0 & 0.43 & 0.43 & 0.43 & 0.45 & 9.6 & 7.7 & 0.52 & 0.53 & 0.52 & 0.53 \\
\hline & & & 390 & 7.4 & 3.8 & 0.92 & 0.92 & 0.92 & 0.93 & 7.2 & 3.4 & 0.90 & 0.91 & 0.90 & 0.92 \\
\hline & & & 3,900 & 7.3 & 3.1 & 0.99 & 0.99 & 0.99 & 0.99 & 6.9 & 3.0 & 0.97 & 0.99 & 0.98 & 0.99 \\
\hline & \multirow[t]{6}{*}{2} & 1 & 39 & 9.3 & 8.0 & 0.47 & 0.58 & 0.52 & 0.58 & 9.2 & 6.4 & 0.30 & 0.44 & 0.40 & 0.44 \\
\hline & & & 390 & 7.2 & 3.5 & 0.76 & 0.89 & 0.84 & 0.89 & 7.2 & 3.5 & 0.56 & 0.90 & 0.76 & 0.91 \\
\hline & & & 3,900 & 7.1 & 3.1 & 0.84 & 0.99 & 0.93 & 0.99 & 6.5 & 2.7 & 0.60 & 0.98 & 0.81 & 0.98 \\
\hline & & 2 & 39 & 9.1 & 6.8 & 0.41 & 0.51 & 0.45 & 0.51 & 9.8 & 6.8 & 0.26 & 0.41 & 0.35 & 0.41 \\
\hline & & & 390 & 7.5 & 3.3 & 0.80 & 0.93 & 0.89 & 0.93 & 6.8 & 3.3 & 0.55 & 0.90 & 0.74 & 0.90 \\
\hline & & & 3,900 & 7.0 & 2.9 & 0.85 & 0.99 & 0.94 & 0.99 & 6.6 & 2.8 & 0.61 & 0.98 & 0.82 & 0.98 \\
\hline $20 \times 20$ & 1 & 1 & 39 & 10.5 & 8.6 & 0.49 & 0.50 & 0.50 & 0.50 & 13.0 & 9.7 & 0.42 & 0.42 & 0.42 & 0.42 \\
\hline & & & 390 & 7.9 & 4.3 & 0.86 & 0.88 & 0.88 & 0.88 & 7.6 & 4.6 & 0.86 & 0.86 & 0.86 & 0.87 \\
\hline & & & 3,900 & 7.4 & 3.7 & 0.97 & 0.99 & 0.98 & 0.99 & 7.3 & 3.8 & 0.98 & 0.98 & 0.98 & 0.99 \\
\hline & & 2 & 39 & 11.9 & 9.0 & 0.47 & 0.49 & 0.48 & 0.49 & 11.6 & 9.6 & 0.40 & 0.41 & 0.40 & 0.41 \\
\hline & & & 390 & 7.8 & 4.8 & 0.88 & 0.89 & 0.89 & 0.89 & 7.9 & 5.1 & 0.88 & 0.89 & 0.88 & 0.89 \\
\hline & & & 3,900 & 7.4 & 3.7 & 0.97 & 0.99 & 0.98 & 0.99 & 7.3 & 3.7 & 0.98 & 0.98 & 0.98 & 0.99 \\
\hline & 2 & 1 & 39 & 10.8 & 10.5 & 0.25 & 0.36 & 0.32 & 0.36 & 12.7 & 9.7 & 0.27 & 0.45 & 0.40 & 0.45 \\
\hline & & & 390 & 8.0 & 5.2 & 0.63 & 0.88 & 0.81 & 0.88 & 9.2 & 4.3 & 0.55 & 0.90 & 0.82 & 0.90 \\
\hline & & & 3,900 & 7.4 & 4.3 & 0.70 & 0.98 & 0.90 & 0.98 & 8.6 & 3.7 & 0.59 & 0.98 & 0.88 & 0.98 \\
\hline & & 2 & 39 & 11.1 & 10.2 & 0.28 & 0.39 & 0.37 & 0.39 & 12.9 & 10.1 & 0.26 & 0.45 & 0.38 & 0.45 \\
\hline & & & 390 & 7.8 & 5.0 & 0.62 & 0.88 & 0.80 & 0.88 & 9.3 & 4.8 & 0.54 & 0.89 & 0.82 & 0.90 \\
\hline & & & 3,900 & 7.4 & 4.2 & 0.70 & 0.98 & 0.90 & 0.98 & 8.6 & 3.5 & 0.59 & 0.98 & 0.88 & 0.98 \\
\hline
\end{tabular}

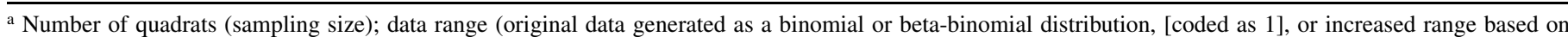
equation 10 in text [coded as 2]); grid index (original distances between quadrats [coded as 1], or distances increased by a factor of 5 [coded as 2]); and number of randomizations in the simulations.

b Table 1 provides explanation of notation and text for description of the four models. 
Since the correlation of $u_{i}$ (and $\left|u_{j}\right|$ ) with $v_{i}$ (and $\left|v_{j}\right|$ ) was generally very high, especially for large sampling sizes, the linear relationship of $\bar{v}_{i}$ and $\left|\bar{v}_{j}\right|$ with $I_{a}$ was nearly identical to that of $\bar{u}_{i}$ with $I_{a}$. The pattern in the differences between $I_{a}$ and $\bar{u}_{i}$ is very similar to that between $\bar{v}_{i}$ and $\left|\bar{v}_{j}\right|$ (Fig. 4A versus Fig. 4B).
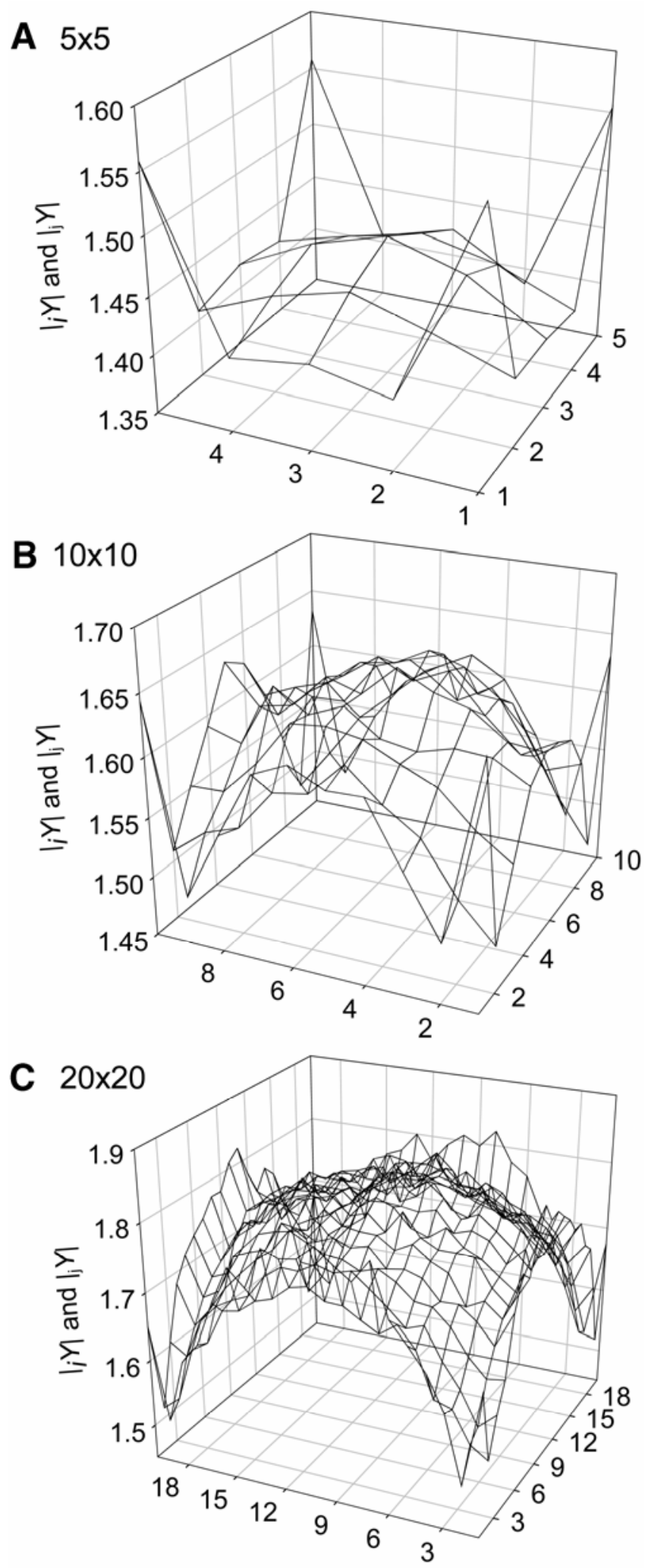

Fig. 2. Plot of spatial analysis by distance indices (SADIE) ${ }_{i} Y$ and $\left|{ }_{j} Y\right|$ against spatial locations for the original beta-binomial data set of $\mathbf{A}, 5 \times 5, \mathbf{B}, 10 \times 10$, and $\mathbf{C}, 20 \times 20$ sampling units, obtained from 3,900 randomizations. Table 1 provides notation and symbols.
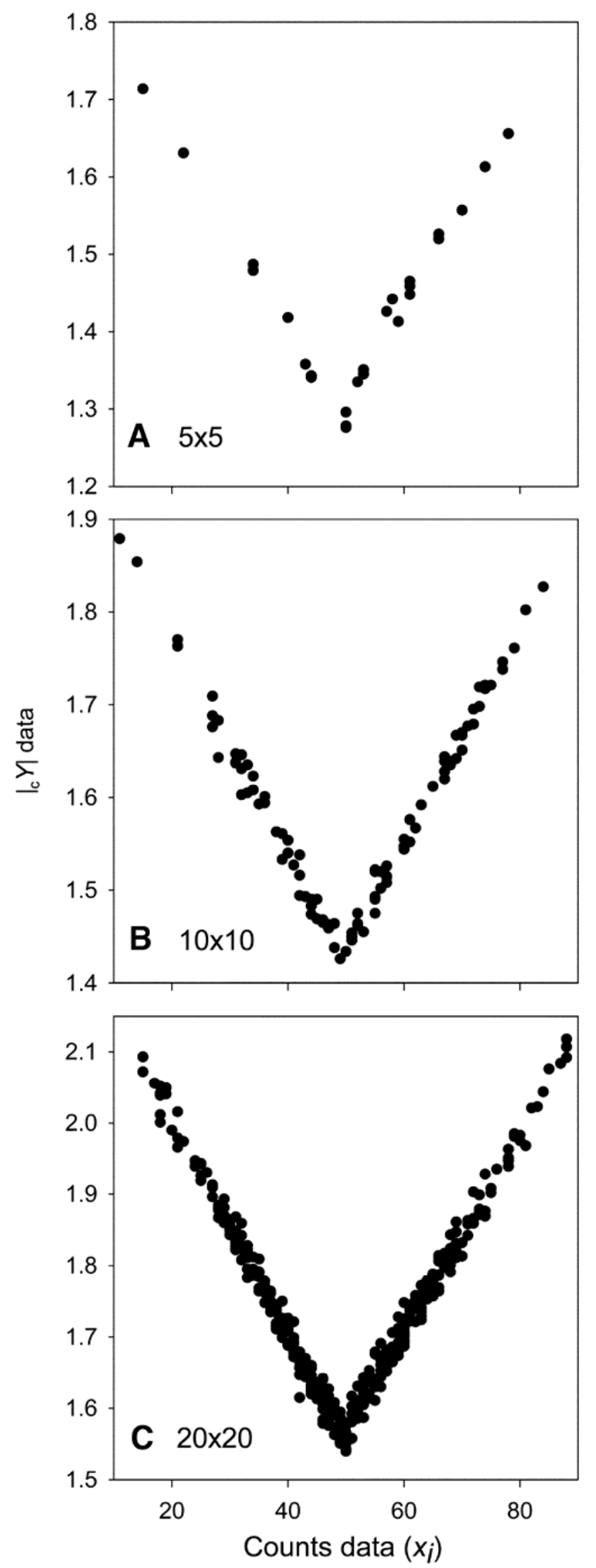

Fig. 3. Plot of spatial analysis by distance indices (SADIE) $\left.\right|_{c} Y \mid$ against $x_{i}$ for the original beta-binomial data set of $\mathbf{A}, 5 \times 5, \mathbf{B}, 10 \times 10$, and $\mathbf{C}, 20 \times 20$ sampling units, obtained from 3,900 randomizations. Table 1 provides notation and symbols. 
The correlation between $\bar{u}_{i}$ and $I_{a}$ increased with increasing sampling size: it was $0.90,0.92,0.98$, and 0.99 for sampling sizes of $3 \times 3,5 \times 5,10 \times 10$, and $20 \times 20$, respectively, which was equal to the correlation between $\left(\bar{v}_{i}+\left|\bar{v}_{j}\right|\right) / 2$ and $I_{a}$ (up to two significant numbers) for the same data sets. The effects of sam-
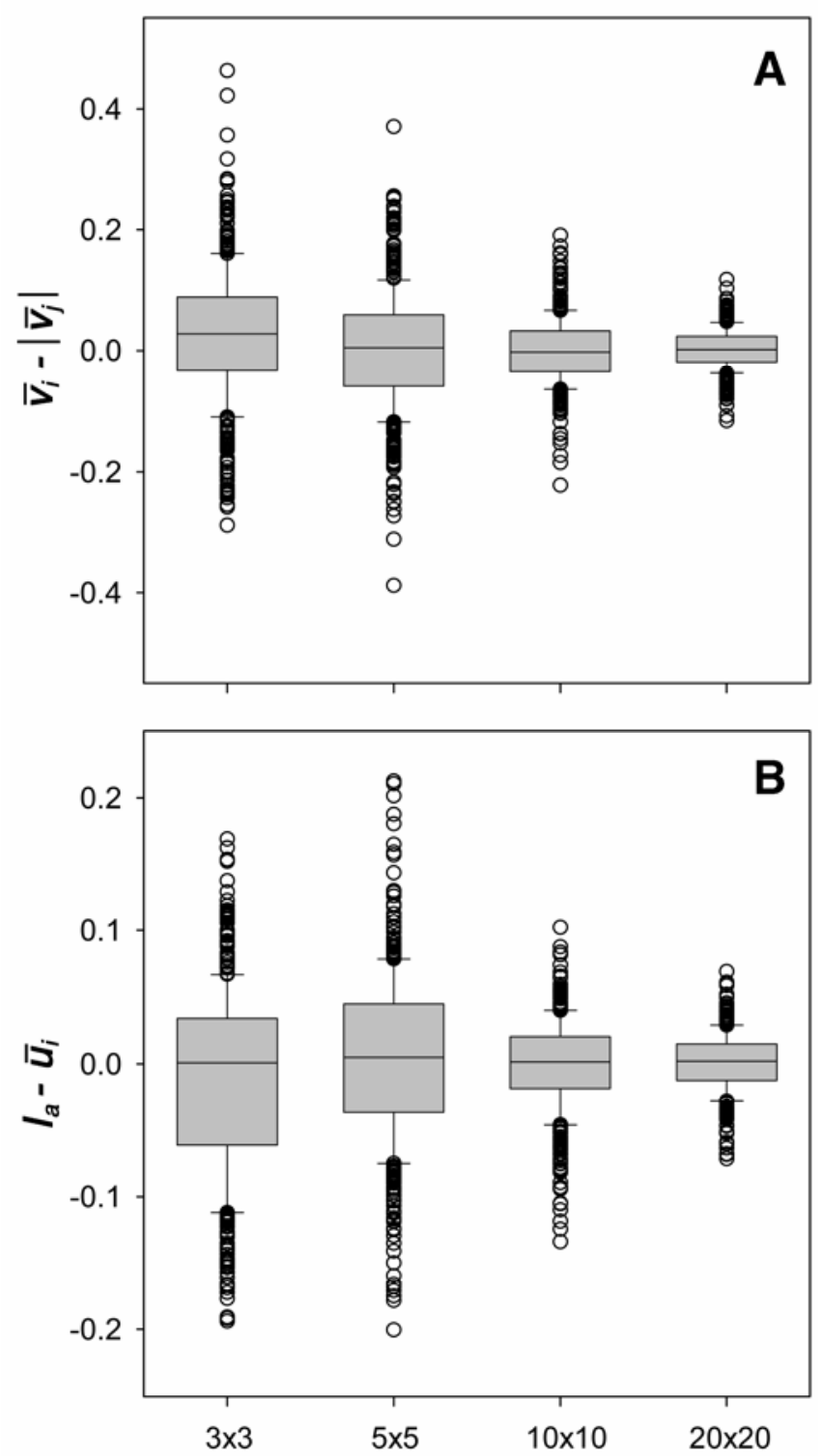

Fig. 4. Boxplots of the differences $\mathbf{A}$, between $\bar{v}_{i}$ and $\left|\bar{v}_{j}\right|$, and $\mathbf{B}$, between $I_{a}$ and $\bar{u}_{i}$. Table 1 provides notation and symbols. The upper and lower limits of the box indicate the upper and lower quartiles of the distribution and the horizontal line through the box indicates the median. The "whiskers' extending beyond the box indicate the range of 10th and 90th percentiles; 'outlying' points are shown individually as open circles. pling size on the relationship among these indices were further confirmed by regression analyses (Table 3 ). The larger the sampling size, the closer the intercept was to zero and the slope was to 1.0. Indeed, for a sampling size of $20 \times 20$, the intercept and slope estimates were not significantly different from 0 and 1.0 , respectively.

Effects of spatial locations on $v_{i}$ and $v_{j}$. Figure 5 shows the cumulative frequency distribution of $Y_{i}$ (or $Y_{j}$ ) corresponding to the three individual counts $\left(x_{i}=78\right.$ [maximum], 52 [ $\sim$ mean], and 15 [minimum]) for four sets of permutations. There were considerable differences among the four distributions for $x_{i}$ values of either 78 or 15 . When $x_{i}=78$, there was the greatest number of large $Y_{i}$ values when this count was at the corner, whereas there was the smallest number of large $Y_{i}$ values when this count was at the center of the grid. The average $Y_{i}$ values were 1.96, 1.62, 1.56, and 1.66 for the locations of $(1,1),(1,3),(3,3)$, and random position, respectively (Table 4); based on pair-wise $t$ tests, all differences were significant except between 1.62 and 1.66. Placing the maximum count at the corner also resulted in greatest variation in $Y_{i}(\mathrm{CV}=39 \%)$, whereas placing it at the center resulted in least variation $(\mathrm{CV}=28 \%)$. Similarly, for $x_{i}=15$, the average $\left|Y_{j}\right|$ values were $2.00,1.68,1.58$, and 1.71 for the locations of $(1,1)$, $(1,3),(3,3)$, and random position, respectively (Table 4). In contrast, for $x_{i}=52$, differences in $Y_{i}$ between the four placement conditions were far less apparent compared with those found for extreme counts of 78 and 15 (Fig. 5). Because $x_{i}=52$ is close to the average of this data set, there are only a few moves to regularity (since $x_{i}-\bar{x}$ is close to 0 ). Thus, $Y_{i}$ can only take a few possible values, and there is a corresponding zig-zag shape (pronounced step-function) to the cumulative distribution. For this count, the average $Y_{i}$ values were $1.38,1.30,1.42$, and 1.35 for the locations of $(1,1),(1,3),(3,3)$, and random position, respectively (Table 4). As expected from the definition of ${ }_{c} Y$, the average value of $Y_{i}$ for the final location treatment (i.e., random location) over the 1,000 permutations was very close to the estimated ${ }_{c} Y$ from the 3,900 randomizations for all three counts (Table 4).

Table 4 shows the summary results of $u_{i}$ and $u_{j}$ and the clustering indices $\left(v_{i}\right.$ and $\left.v_{j}\right)$ calculated from the $Y_{i}\left(Y_{j}\right)$ values presented in Figure 5. The $u_{i}\left(u_{j}\right)$ value (hence $v_{i}$ and $v_{j}$ ) was influenced by both counts and spatial location. Because the 1,000 $Y_{i}$ (or $Y_{j}$ ) in Figure 5, as well as $v_{i}$ (or $v_{j}$ ) values were from randomly permutated data sets (with one value being fixed), these permutations can be considered a random sample of the population of spatial patterns where the specific count was held at the specific location in relation to all possible combinations of all other counts in the remaining locations. The specific spatial patterns for each permutation range from regular to highly aggregated, but will be expected to be random on average. The principle of the SADIE methodology is that a value of an index $\left(v_{i},\left|v_{j}\right|, \bar{v}_{i}\right.$ and $\left.\left|\bar{v}_{j}\right|, I_{a}\right)$ close to unity indicates a random pattern, because the index is a ratio between an observed (local or global) distance value (or its scaled version) and an average value across randomizations. It follows that for each count the average $v_{i}$ and $v_{j}$ over the 1,000 random permutations should be close to 1.0 over the 1,000

TABLE 3. Summary of regression analyses of $\bar{v}_{i}$ on $\left|\bar{v}_{j}\right|$, and of $I_{a}$ on $\left(\bar{v}_{i}+\left|\bar{v}_{j}\right|\right) / 2^{\mathrm{a}}$

\begin{tabular}{|c|c|c|c|c|c|c|}
\hline \multirow[b]{2}{*}{ Sampling size } & \multicolumn{3}{|c|}{$\bar{v}_{i}$ on $\left|\bar{v}_{j}\right|^{\mathrm{b}}$} & \multicolumn{3}{|c|}{$I_{a}$ on $\left(\bar{v}_{i}+\left|\bar{v}_{j}\right|\right) / 2^{\mathrm{b}}$} \\
\hline & Intercept & Slope & $R_{a}^{2}$ & Intercept & Slope & $R_{a}^{2}$ \\
\hline $3 \times 3$ & $0.278(0.037)$ & $0.698(0.036)$ & 0.42 & $-0.147(0.026)$ & $1.158(0.026)$ & 0.80 \\
\hline $5 \times 5$ & $0.214(0.027)$ & $0.782(0.027)$ & 0.63 & $-0.042(0.020)$ & $1.039(0.020)$ & 0.85 \\
\hline $20 \times 20$ & $0.028(0.011)$ & $0.970(0.011)$ & 0.94 & $\ldots^{\mathrm{c}}$ & $0.999(0.001)$ & 0.97 \\
\hline
\end{tabular}

a The data used for the analysis are shown in Figures 4 and 5 and generated using the original beta-binomial distribution data sets. Table 1 provides explanation of notation.

${ }^{\mathrm{b}}$ Linear regression model. Numbers in parentheses are standard errors of intercepts and slopes. The percentage of variance accounted for by the model is summarized as $R_{a}^{2}$ (the coefficient of determination adjusted for degrees of freedom).

c The intercept is not significantly different from zero and hence set to zero when fitting the model. 
permutations. For all three counts, the average values of $v_{i}$ and $v_{j}$ were close to this expected value of 1.0 only when the point was held at the middle of an edge, but for the other two positions they deviated considerably from 1.0 (Table 4). This deviation appeared to increase as the count concerned was further away from the mean.

\section{DISCUSSION}

SADIE methodology has been widely and successfully used recently in describing and quantifying spatial count data in several disciplines, including plant pathology $(1-5,11,12,14,15)$. Although Perry et al. (10) now recommend the use of $v_{i^{-}}$and $v_{j}$-based statistics for quantifying patterns in addition to the $I_{a}$ statistic, it is still common for others to simply report $I_{a}$ (and associated significance level). In assessing the results from several experimental data sets $(11,12)$ and from a spatial analysis of predictions from a stochastic epidemic simulation model (17), a close correlation between $\bar{v}_{i},\left|\bar{v}_{j}\right|$, and $I_{a}$ was observed. Here we have shown that $\bar{v}_{i}$ and $\left|\bar{v}_{j}\right|$ are mathematically related to $I_{a}$. Both $\bar{v}_{i}$ and $\left|\bar{v}_{j}\right|$ are weighted averages of individual $I_{a}$ values (i.e., $u_{i}$; equations 4 and 5 ), with the weight proportional to $1 /_{i} Y$, and $I_{a}$ is the ratio of the unweighted mean numerator and denominator of the $u_{i}$ values, which will approximately equal the mean of $u_{i}$, depending on the exact configuration of average distance moved for each counts. This is indeed shown to be the case in several artificially generated data sets. This relationship improved as sampling size increased. Thus, both types of statistics are essentially sums or averages of the distance moved by individuals for each sampling unit to achieve a regular distribution, but measured at two different scales: $I_{a}$ on the whole sampling grid, whereas $\bar{v}_{i}$ and $\left|\bar{v}_{j}\right|$ (or $u_{i}$ ) on individual sampling units.

We have shown that ${ }_{c} Y$ (a component of $v_{i}$ and $v_{j}$; equation 4) had a near-perfect linear relationship with $x$ (the original counts) using a limited number of data sets, although this relationship for outflow units may be different from inflow units. A common relationship may exist between ${ }_{c} Y$ and $x$ for both inflow and outflow units if counts data are approximately symmetrical, i.e., for those data sets generated with either a Poisson or a beta-binomial distribution about the mean irrespective of their spatial locations. Further studies are needed to investigate the applicability of this linear relationship. Theoretically, ${ }_{c} Y$ is strictly a function of $x$ for a given data set (i.e., for a given frequency distribution). Thus, identical counts within a given data set should result in identical ${ }_{c} Y$ values. However, due to insufficient randomizations (for instance, even with a $3 \times 3$ lattice, there are 9 ! [ $[=362,880]$ possible arrangements of nine unique counts), we found that calculated ${ }_{c} Y$ values for identical counts (within a single data set) were usually different, albeit the differences were often very small with the SADIE algorithm. This anomaly could be removed by using ${ }_{c} Y$ values estimated from its relationship with $x$, which is derived from the ${ }_{c} Y$ values from randomizations. This is the main justification for estimating ${ }_{c} Y$ from the counts rather than using ${ }_{c} Y$ values directly from the randomizations in some of this study.

Perry et al. (10) made a highly significant advance in the SADIE approach to spatial analysis by quantifying the contribution of each sampling unit to the pattern through the average distance index, $Y_{i}$ (or $Y_{j}$ ). The collection of these indices for a data set can be used directly to determine the pattern to the organism under investigation (Figs. 4 and 5 in Perry et al. [10]). However, Perry et al. (10) pointed out the value of obtaining standardized and unitless indices for each sampling unit $\left(v_{i}\right.$ or $\left.v_{j}\right)$. Equation 2 is one way of obtaining this standardized index, based on the global averages ${ }_{i} Y$ and ${ }_{c} Y$. Using several artificially generated data sets, we have shown that the variation of the location-dependent weight ${ }_{i} Y$ ) used to calculate $v_{i}$ and $v_{j}$ was generally small (its $\mathrm{CV}$ was generally $<4 \%$ ). Particularly, the units near the corners and on the
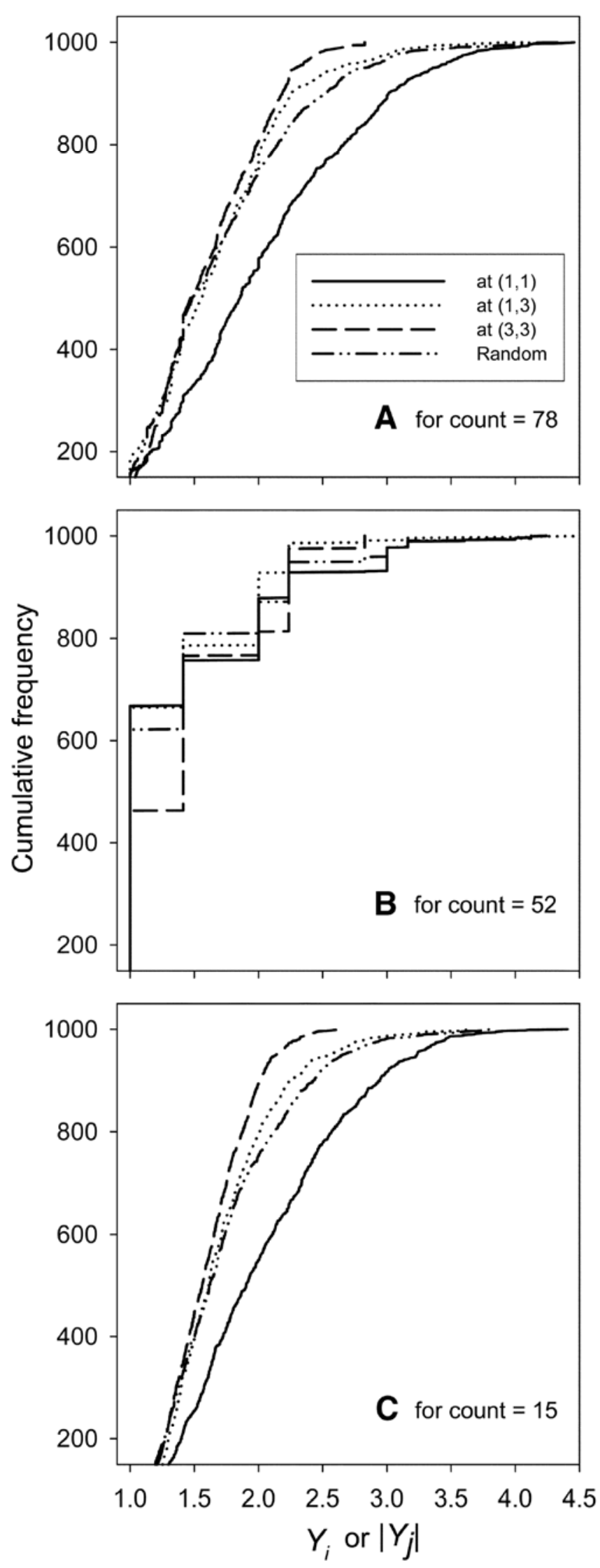

Fig. 5. Empirical cumulative distributions of $Y_{i}$ or $\left|Y_{j}\right|$, where the count $\mathbf{A}, x_{i}=$ 78 (maximum in data set), $\mathbf{B}, x_{i}=52$ (close to the mean), and $\mathbf{C}, x_{i}=15$ (minimum) of the original beta-binomial $5 \times 5$ data set was either fixed at one of three positions, corner, edge, and center, or allowed to move in all randomizations (the latter being the standard approach). For each case, 1,000 randomizations were done. Table 1 provides notation and symbols. 
edge appeared to have lower and higher ${ }_{i} Y$ values, respectively, especially for large sampling sizes. Hence, in calculating $v_{i}$ or $v_{j}$, more weight is given to the sampling units near the corners (but not necessarily exactly at the corner) and less to other units on the edge.

On the basis of the results obtained here, we suggest that use of ${ }_{i} Y$ in determining a location-specific clustering index such as $v_{i}$ (or $v_{j}$ ) may be unnecessary or undesirable. First, nonweighted clustering indices (i.e., $u_{i}$ ) were highly correlated with weighted indices (i.e., $v_{i}$ or $v_{j}$ ) even for a sampling size as small as $5 \times 5$. These two types of indices were virtually identical for sampling sizes of $10 \times 10$ and $20 \times 20$ (correlation coefficient $>0.99$ ). This is because sampling units at or near the corners and edges were the only locations that had a discernible (or consistent) effect on ${ }_{i} Y$, and these locations comprise only a small-to-moderate percentage of the total for large sampling areas. However, there were considerable differences between the weighted and unweighted statistics in some permutations for the sampling size of $3 \times 3$. This is because the edges and corners comprised a large proportion of the total for such a small sampling size; eight out of the total nine sampling units are on the edges and corners. However, it is very unlikely that in practice we would use a sampling size as small as $3 \times 3$. Furthermore, at all sampling sizes, even for $3 \times 3$, $I_{a}$ was highly correlated with $\bar{v}_{i}$ and $\left|\bar{v}_{j}\right|$, as well as with $\bar{u}_{i}$. Second, average $\mathrm{CV}$ of ${ }_{i} Y$ was about $3.6 \%$. Assuming a normal distribution for ${ }_{i} Y, 95 \%$ of the values are within $7.2 \%$ of the mean. Therefore, for most sampling units, $1.08>w_{i}=\left({ }_{o} Y l_{i} Y\right)>0.93$, close to 1 . Therefore for larger sampling sizes, $u_{i}$ is nearly identical to $v_{i}\left(v_{j}\right)$. If standardization was done without reliance on ${ }_{i} Y$, such as the use of $u_{i}$ (equation 5) for the location-specific index, the relationship of $I_{a}$ with the location-specific indices would be easier to interpret. Namely, the indices all measure generally the same phenomenon, relative distance to regularity, but at different scales. $I_{a}$ measures the relative distance to regularity for the whole sampling grid (a global index), whereas $u_{i}$ values measure relative distance to regularity for each individual sampling unit.

When there is a large difference between $\bar{v}_{i}$ and $\left|\bar{v}_{j}\right|$ in an observed data set, it is important to know the biological, ecological, and/or physical reasons for such a difference. Our investigation indicated the large difference in $\bar{v}_{i}$ and $\left|\bar{v}_{j}\right|$ can result from sampling sizes: the difference increases with decreasing sizes. This may result from a high number of sampling units in corners and on edges relative to those in interior units for small sampling sizes based on simple geometric principles. Hence, it is more probable to have a situation where most inflow or outflow units are in the interior region, or on the edges, or near/on the corner for a given small data set. Of course, such an extreme spatial arrangement may be due to some biological/ecological factors. This may lead to a large difference in $\bar{v}_{i}$ and $\left|\bar{v}_{j}\right|$ due to differences in ${ }_{i} Y$ and $Y_{i}$. For larger sampling sizes, the number of sampling units at or near corners and on edges relative to those interior units is small and, hence, their effects may be expected to be relatively less important. Hence, we suggest that a large difference in $\bar{v}_{i}$ and $\left|\bar{v}_{j}\right|$ is less likely due to biological or ecological factors for small sampling sizes than for large sampling sizes where edge/corner effects are not dominating the results.

The individual clustering indices of SADIE are defined and calculated as standardized $Y_{i}$ values in an attempt to take into account independently the potential effects of the spatial location of counts, in the form of ${ }_{i} Y$, and the actual count itself, in the form of ${ }_{c} Y$. However, results from a few selected examples indicated that the effects of the spatial location and the count itself on $Y_{i}$ are not independent. ${ }_{i} Y$ did not adequately account for the location effects since the average $v_{i}$ and $v_{j}$ values for the three selected points deviated considerably from the expected value of 1.0 for the corner and center positions. Moreover, the effect of spatial location of the count on $Y_{i}$ varied with the count itself. This tendency of overestimating clustering indices might partially explain the observation that the test based on the clustering indices can be much more powerful at detecting nonrandomness than the overall index $I_{a}$, especially for situations with large counts around the boundaries of the sampled area (10). It may also partially explain the finding that mean clustering indices are sensitive to absolute patch positions (16). We conclude that $v_{i}$ (or $v_{j}$ ) and $u_{i}$ (or $u_{j}$ ), as currently defined, do not fully account for spatial-location effects on clustering because of the interaction of spatial location and count values on the ${ }_{i} Y$ statistic. Further research is needed to explore possible alternative standardization methods for the local clustering index. Because of the nature of the SADIE approach, any alternative will require intensive computation.

In summary, we have arrived at several conclusions regarding the interrelationships between $I_{a}, \bar{v}_{i}$, and $\left|\bar{v}_{j}\right|$, as well as between the indices for the individual sampling units $\left(u_{i}, v_{i}\right.$, and $\left.v_{j}\right)$, based on artificially generated data sets and demonstrated mathematical relationships in the current study and the interpretation of results from our previous investigations $(16,17)$. First, we have suggested that it may not be necessary to use ${ }_{i} Y$ when calculating a scaled index for each sampling unit such as $v_{i}$ and $v_{j}$. Second, we conclude that differences in $\bar{v}_{i}$ and $\left|\bar{v}_{j}\right|$, if any, are critically affected by sampling sizes. Third, except for very small sampling grids, there is little difference in results based on $I_{a}$, and $\bar{v}_{i}$ (or $\left.\left|\bar{v}_{j}\right|\right)$.

TABLE 4. Summary of $Y_{i}$ (or $\left|Y_{j}\right|$ ) and $v_{i}$ (or $\left|v_{j}\right|$ ) for counts of 78, 52, and 15 either fixed at three different positions, or allowed to vary randomly over 1,000 permutations of the original $5 \times 5$ data set generated from a beta-binomial distribution ${ }^{\mathrm{a}}$

\begin{tabular}{|c|c|c|c|c|c|c|c|c|c|}
\hline \multirow[b]{2}{*}{ Counts } & \multirow[b]{2}{*}{ Position } & \multirow[b]{2}{*}{$\bar{Y}_{i}$} & \multicolumn{3}{|c|}{$u_{i}=Y_{i} /{ }_{c} Y$} & \multirow[b]{2}{*}{${ }_{i} Y^{\mathrm{b}}$} & \multicolumn{3}{|c|}{$v_{i}=w_{i} u_{i}=\left({ }_{o} Y /{ }_{i} Y\right) u_{i}$} \\
\hline & & & Minimum & Mean & Maximum & & Minimum & Mean & Maximum \\
\hline \multirow[t]{4}{*}{$78\left({ }_{c} Y=1.656\right)^{\mathrm{c}}$} & Corner & 1.957 & 0.604 & 1.182 & 2.693 & 1.574 & 0.555 & 1.087 & 2.476 \\
\hline & Edge & 1.623 & 0.604 & 0.980 & 2.460 & 1.423 & 0.614 & 0.997 & 2.502 \\
\hline & Center & 1.563 & 0.604 & 0.944 & 1.708 & 1.471 & 0.594 & 0.929 & 1.680 \\
\hline & Random & 1.658 & 0.604 & 1.001 & 2.616 & $\ldots$ & $\ldots$ & $\ldots$ & $\ldots$ \\
\hline \multirow[t]{4}{*}{$52\left({ }_{c} Y=1.335\right)$} & Corner & 1.375 & 0.749 & 1.030 & 3.178 & 1.574 & 0.689 & 0.947 & 2.922 \\
\hline & Edge & 1.296 & 0.749 & 0.971 & 2.724 & 1.423 & 0.762 & 0.987 & 2.770 \\
\hline & Center & 1.419 & 0.749 & 1.063 & 2.118 & 1.471 & 0.737 & 1.046 & 2.083 \\
\hline & Random & 1.347 & 0.749 & 1.009 & 3.088 & $\ldots$ & $\ldots$ & $\ldots$ & $\ldots$ \\
\hline \multirow[t]{4}{*}{$15\left({ }_{c} Y=1.714\right)$} & Corner & 2.002 & 0.583 & 1.168 & 2.571 & 1.574 & 0.536 & 1.074 & 2.364 \\
\hline & Edge & 1.682 & 0.583 & 0.981 & 2.122 & 1.423 & 0.593 & 0.998 & 2.158 \\
\hline & Center & 1.578 & 0.583 & 0.921 & 1.535 & 1.471 & 0.574 & 0.906 & 1.510 \\
\hline & Random & 1.712 & 0.583 & 0.999 & 2.223 & $\ldots$ & $\ldots$ & $\ldots$ & $\ldots$ \\
\hline
\end{tabular}

a Table 1 provides notation and full explanation of symbols.

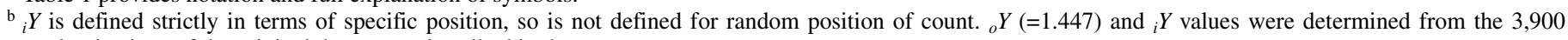
randomizations of the original data set as described in the text.

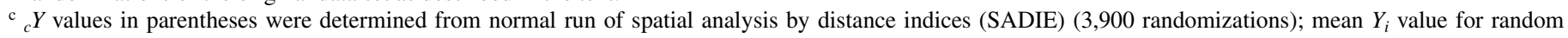
position of count was based on the 1,000 randomizations and is equivalent to the ${ }_{c} Y$ in parentheses. 
Finally, in interpreting SADIE indices and comparing them between different studies, investigators must keep in mind that the magnitudes of $I_{a}$, and $\bar{v}_{i}$ (or $\left|\bar{v}_{j}\right|$ ), are more influenced by the absolute sampling position and initial epidemic conditions (number of initial infected plants and their spatial configuration) than with spore dispersal gradients (i.e., length scale over which disease spread occurs) or other ecological/biological processes occurring during the epidemics $(16,17)$. We have shown here that the current method for calculating clustering indices does not completely adjust for the effects of the actual counts and their sampling positions. Thus, we can conclude that differences in the calculated SADIE indices, as currently defined, between different data sets are probably mostly due to nonbiological factors or processes, such as sampling scheme used, and initial conditions. Direct quantitative comparison of the magnitude of indices should be restricted to data sets with similar sampling schemes and initial conditions (e.g., physical position of the original patches of disease). We agree with Perry et al. (10) regarding the value of a local clustering index for characterizing patterns of organisms, including diseased plants. The $v_{i}$ (or $v_{j}$ ) index, however, is not the only possible scaled metric for representing clustering at a particular location. We thus believe, as discussed above, that alternative definitions of a unitless local clustering index, taking into account the effects of the interactions between the spatial location and the counts itself on the distance to regularity, are warranted. Research on alternative local clustering indices in comparison to the current one would ultimately indicate the degree of statistical bias that may exist in the current approach to quantifying the clustering at each location, as well as the conditions that lead to low or high bias. It should be noted, however, that bias is not by itself a problem in analysis, if the magnitude of the bias is known or can be estimated.

\section{ACKNOWLEDGMENTS}

We thank J. B. Scott and S. J. Pethybridge for sharing their results for $I_{a}, \bar{v}_{i}$, and $\left|\bar{v}_{j}\right|$ with us.

\section{LITERATURE CITED}

1. Dungan, J. L., Perry, J. N., Dale, M. R. T., Legendre, P., Citron-Pousty, S., Fortin, M. J., Jakomulska, A., Miriti, M., and Rosenberg, M. S. 2002. A balanced view of scale in spatial statistical analysis. Ecography 25:626-640.

2. Holland, J. M., Winder, L., and Perry, J. N. 2000. The impact of dimethoate on the spatial distribution of beneficial arthropods in winter wheat. Ann. Appl. Biol. 136:93-105.

3. Kleijn, D., and Steinger, T. 2002. Contrasting effects of grazing and hay cutting on the spatial and genetic population structure of Veratrum album, an unpalatable, long-lived, clonal plant species. J. Ecol. 90:360-370.

4. Korie, S., Perry, J. N., Mugglestone, M. A., Clark, S. J., Thomas, C. F. G., and Roff, M. N. M. 2000. Spatiotemporal associations in beetle and virus count data. J. Agric. Biol. Environ. Stat. 5:214-239.

5. Maestre, F. T. 2003. Small-scale spatial patterns of biological crusts in a Mediterranean semi-arid ecosystem. Rev. Chilena Historia Nat. 76:35-46.

6. Payne, R. (ed.) 2002. The Guide to GenStat Release 6.1-Part 2: Statistics. VSN International, Oxford.

7. Perry, J. N. 1995. Spatial-analysis by distance indexes. J. Animal Ecol. 64:303-314.

8. Perry, J. N. 1998. Measures of spatial pattern for counts. Ecology 79:1008-1017.

9. Perry, J. N., and Dixon, P. M. 2002. A new method to measure spatial association for ecological count data. Ecoscience 9:133-141.

10. Perry, J. N., Winder, L., Holland, J. M., and Alston, R. D. 1999. Red-blue plots for detecting clusters in count data. Ecol. Letts. 2:106-113.

11. Pethybridge, S. J., and Turechek, W. W. 2003. Analysis of the association among three viruses infecting hop in Australia. Plant Pathol. 52:158-167.

12. Scott, J. B., Hay, F. S., Wilson, C. R., Cotterill, P. J., and Fist, A. J. 2003. Spatiotemporal analysis of epiphytotics of downy mildew of oilseed poppy in Tasmania, Australia. Phytopathology 93:752-757.

13. Turechek, W. W., and Madden, L. V. 1999. Spatial pattern analysis of strawberry leaf blight in perennial production systems. Phytopathology 89:421-433.

14. Turechek, W. W., and Madden, L. V. 2000. Analysis of the association between the incidence of two spatially aggregated foliar diseases of strawberry. Phytopathology 90:157-170.

15. Winder, L., Alexander, C. J., Holland, J. M., Woolley, C., and Perry, J. N. 2001. Modelling the dynamic spatio-temporal response of predators to transient prey patches in the field. Ecol. Letts. 4:568-576.

16. Xu, X.-M., and Madden, L. V. 2003. Considerations for the use of SADIE statistics to quantify spatial patterns. Ecography 26:821-830.

17. Xu, X.-M., and Madden, L. V. 2004. Use of SADIE statistics to study spatial dynamics of plant disease epidemics. Plant Pathol. 53:38-49. 